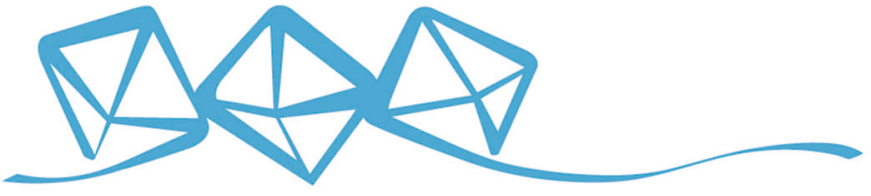 COMMUNICATIONS MATERIALS
}

\section{Controlled steric selectivity in molecular doping towards closest-packed supramolecular conductors}

\author{
Shinya Kohno', Yu Yamashita',2, Naotaka Kasuya ${ }^{1}$, Tsubasa Mikie ${ }^{3}$, Itaru Osaka ${ }^{3}$, Kazuo Takimiya ${ }^{4,5}$, \\ Jun Takeya ${ }^{1,2,6} \&$ Shun Watanabe $e^{1,6,7 凶}$
}

Recent developments in molecular doping technologies allow extremely high carrier densities in polymeric semiconductors, exhibiting great diversity because of the unique size, conformation, and steric effect of molecular dopants. However, it is controversial how steric effects can limit the doping efficiency and to what extent dopants can be accommodated in polymers. Here, we employ two distinct conjugated polymers with different alkyl side-chain densities, where polymers are doped via anion-change, allowing greater variation in the incorporation of molecular dopants having different electrostatic potentials and shapes. We characterize the doping efficiency with regard to steric effects, considering the unique void space in the conjugated polymers. Our study reveals that doping efficiency of polymers with sparse alkyl side-chains is significantly greater than that with dense side-chains. A closestpacked supramolecule is realized with a particular combination of a sparse polymer and a large dopant, giving rise to high conductivity, air stability, and remarkably high work function. This work provides a critical insight into overcoming steric effects in molecular doping.

\footnotetext{
${ }^{1}$ Material Innovation Research Center (MIRC) and Department of Advanced Materials Science, Graduate School of Frontier Sciences, The University of Tokyo, 5-1-5 Kashiwanoha, Kashiwa, Chiba 277-8561, Japan. ${ }^{2}$ International Center for Materials Nanoarchitectonics (WPI-MANA), National Institute for Materials Science (NIMS), 1-1 Namiki, Tsukuba 205-0044, Japan. ${ }^{3}$ Department of Applied Chemistry, Graduate School of Engineering, Hiroshima University, 1-4-1 Kagamiyama, Higashi-Hiroshima, Hiroshima 739-8527, Japan. ${ }^{4}$ Department of Chemistry, Graduate School of Science, Tohoku University, 6-3 Aoba, Aramaki, Aoba-ku, Sendai, Miyagi 980-8578, Japan. ${ }^{5}$ Emergent Molecular Function Research Team, RIKEN Center for Emergent Matter Science (CEMS), 21 Hirosawa, Wako, Saitama 351-0198, Japan. ${ }^{6}$ AIST-UTokyo Advanced Operando-Measurement Technology Open Innovation Laboratory (OPERANDOOIL), National Institute of Advanced Industrial Science and Technology (AIST), 5-1-5 Kashiwanoha, Kashiwa, Chiba 277-8561, Japan. ${ }^{7}$ JST PRESTO, 4-1-8 Honcho, Kawaguchi, Saitama 332-0012, Japan. ${ }^{凶}$ email: swatanabe@edu.k.u-tokyo.ac.jp
} 
1 mpurity doping is one of the most important semiconductor processes, by which (opto)electronic properties can be tuned precisely $^{1,2}$. A dopant, that is, either an electron donor or an acceptor, is incorporated into a semiconducting medium and can shift the Fermi energy. The concept of impurity doping, already established in silicon electronics, has been extended to organic semiconductors (OSCs), where molecular dopants rather than atomic dopants are important agents in chemical reactions ${ }^{3-8}$. Various dopants and processes have been utilized to achieve efficient chemical doping of OSCs, particularly for p-type doping. Here, an integer electron transfer occurs when a redox reaction between a $\pi$-conjugated polymer and a dopant is energetically favorable, resulting in a donor-acceptor association. Recently, relatively high doping levels in polymeric semiconductors have been realized via various molecular doping methods, such as chemical engineering utilizing self-compensation by covalent bounded counter-ions ${ }^{9-11}$, photo-doping ${ }^{12}$, and solid-state diffusion $^{13-16}$, as well as possibly via double doping ${ }^{17}$. Of these methods, extremely high carrier density with the charge density per unit volume of $10^{21} \mathrm{~cm}^{-3}$ in polymeric semiconductors can be achieved via anion-exchange doping ${ }^{18,19}$. In anion-exchange doping, a host-guest system also includes a large excess of a salt (consisting of a cation, and an anion). A conventional p-type doping is initiated by an acceptor dopant (e.g., 2,3,5,6-tetrafluoro7,7,8,8-tetracyanoquinodimethane; F4TCNQ), resulting in the formation of intermediate [polmyer ${ }^{\bullet}{ }^{+} 4 T_{C N Q}{ }^{-}$]. The second anions that are supplied by the additional salt are instantaneously exchanged with F4TCNQ ${ }^{\bullet-}$. The intermediate consisting of F4TCNQ is exchanged to the secondary ion pair via anion exchange, where the intermediate is likely to undergo full integer charge transfer. Anion-exchange doping uses ionic interactions as a driving force to promote doping levels, and allows an anionexchange efficiency of nearly $100 \%$ when an optimum salt is used. A critical difference of anion-exchange doping from conventional molecular doping is that the resulting dopant incorporated in the $\pi$-conjugated polymers does not necessarily initiate redox reaction; the initial electron transfer can be triggered by conventional, redox-active dopants, and the anion exchange process is then followed to form the secondary donor-acceptor pair. This functional separation may facilitate a novel host-guest structure that has not been achieved by conventional doping, suggesting that there are opportunities to explore functional host-guest systems, which will allow a greater variation of molecular dopants having different electrostatic potentials/shapes to be incorporated into polymeric semiconductors. In particular, steric effects of molecular dopants in hybrid host-guest systems would be investigated systematically.

Until now, high doping levels have been achieved only to a limited extent in semicrystalline, polymeric semiconductors ${ }^{13-16,18,19}$. This may be because spatially confined donor-acceptor association is only established within restricted regions in semicrystalline polymers ${ }^{13-15}$, where molecular dopants are intercalated specifically into nanometersized voids that are likely to be alkyl side-chain regions. The most critical factor driving molecular doping is likely to be the energy gain due to redox reactions between the host $\pi$-conjugated polymer and the guest dopant, though structural factors, such as the unique size, conformation, and steric effect of molecular dopants in hybrid host-guest systems, may cause apparent limitations in the maximum achievable doping level ${ }^{20-22}$. However, such steric effects associated with the donor-acceptor association have not been investigated systematically in polymeric semiconductors because of the limited variations of host-guest systems.

In this work, we employ two distinct conjugated polymers with different alkyl side-chain densities, and characterize the doping efficiency regarding steric effects. Spectroscopic and magnetotransport measurements reveal that doping efficiency of polymers with sparse alkyl side-chains is significantly greater than that with dense side-chains. Steric effects between the $\pi$-conjugated semiconducting polymer and dopants could influence the donor-acceptor formation, which strongly limits the maximum achievable doping level in polymeric semiconductors. A closestpacked supramolecule is realized with a particular combination of a sparse polymer and a large dopant, giving rise to high conductivity, air stability, and remarkably high work function.

\section{Results and discussion}

Difference in doping efficiency due to steric effects. We focus on two distinct $\pi$-conjugated polymers, based on PNDTBT (poly (2,7-bis(3-alkylthiophen-2-yl)naphtho[1,2-b:5,6- $\left.b^{\prime}\right]$ dithiophene; Fig. $1 \mathrm{a}, \mathrm{b})^{23-26}$. PNDTBT is a series of naphthodithiophenebithiophene polymers that shows an excellent field-effect mobility up to $1 \mathrm{~cm}^{2} \mathrm{~V}^{-1} \mathrm{~s}^{-1}$ due to its promoted, twodimensional lamellar structure ${ }^{24}$. Specifically, two PNDTBT polymers with different alkyl side-chain densities are employed, PNDTBT-C20, with a sparse density, and PNDTBT-4C16, with a high density. PNDTBT-4C16 possesses four alkyl side-chains in a repeat unit, which clearly limits the size of the void space between each lamella. Space-filling, van der Waals-based representations of PNDTBT-C20 and PNDTBT-4C16 are shown in Figs. 1c, d. The characteristic void distance $d_{\text {void }}$ is defined as the distance of the van der Waals surfaces between alkyl side-chains (Fig. 1c, d).

The molecular doping was performed via anion exchange ${ }^{18}$. In the anion-exchange doping, a solid-state thin film of PNDTBT was immersed in a dopant solution. Here, F4TCNQ and lithiumbis(trifluoromethylsulfonyl)imide $\left(\left[\mathrm{Li}^{+} \mathrm{TFSI}^{-}\right]\right)$salt were codissolved in $n$-butyl acetate (see experimental details in the "Methods" section and Supplementary Information Section 1.1). The molecular doping proceeds with an initiator molecule F4TCNQ possibly via a charge transfer interaction. The F4TCNQ radical anions, which are part of the solid-state, donor-acceptor pairs [PNDTBT ${ }^{\bullet}{ }^{+} 4 T_{C N Q}{ }^{\bullet}$ ], are then replaced instantaneously by TFSI $^{-}$via anion exchange (Fig. 1e ${ }^{18}$. Anion-exchange doping allows both efficient molecular doping with an anion-exchange efficiency close to $100 \%$ and a wider variation of functional dopants to be incorporated into polymeric semiconductors. The molecular doping is efficient because the donor-acceptor association minimizes the Gibbs free energy, where the energy gain to form the donor-acceptor pair is believed to be governed predominantly by the redox potential, described by Marcus theory ${ }^{27}$. The anion exchange process utilizes an ionic interaction as an additional driving force in doping; an additional energy gain is expected due to a secondary donor-acceptor association via an anion exchange. Consequently, remarkably high doping levels, close to the half-filled state, can be achieved.

We characterize the doping efficiency with regard to the steric effects related to the unique void space of dopant anions in these two PNDTBT polymers. In the previous literature ${ }^{24}$, it was concluded that both polymers have an extended, planar conformation, which has been verified by X-ray diffraction (XRD), optical absorption measurements, and density functional theory (DFT) calculation. The backbone coplanarity and ordering for the PNDTBT-4C16 is slightly enhanced compared to the PNDTBT-C20. This is presumably because the additional alkyl groups on the naphthodithiophene core can fill the space between the alkyl groups on the thiophene rings which sandwich the naphthodithiophene core. Schematic illustrations of the lamellar structure of PNDTBT polymers are shown in Fig. 2a, b, where the red dotted squares denote the alkyl side-chain regions. We also performed DFT calculations to illustrate the van der Waals surfaces (Fig. 2c-f). Initially, the conformation of a monomer unit 
a

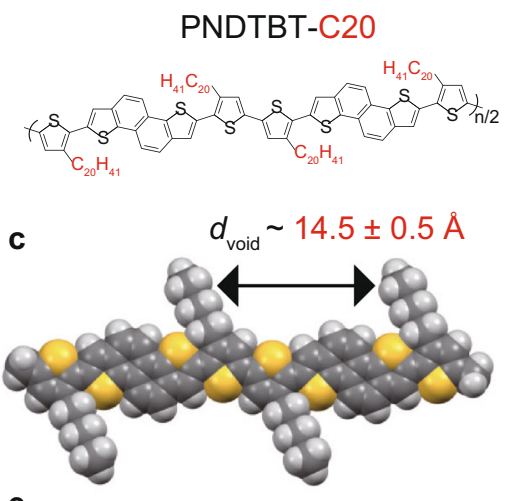

b
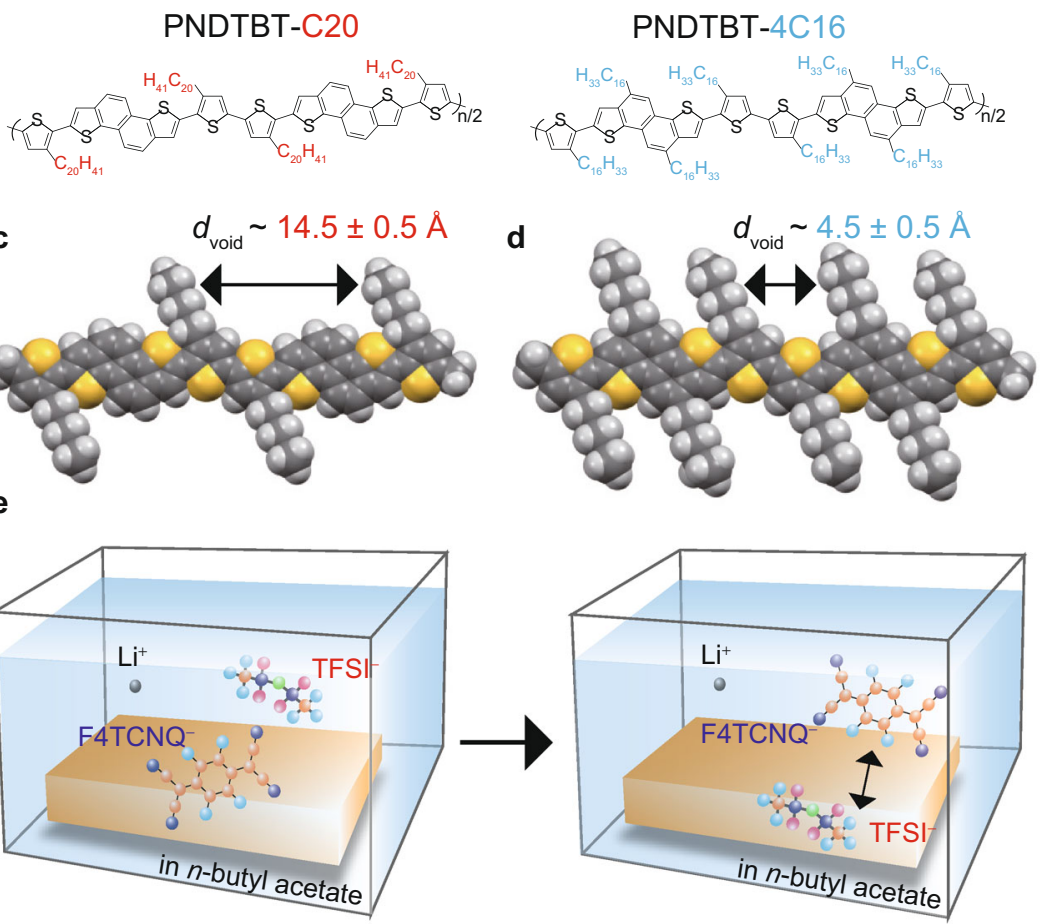

$\left[\mathrm{PNDTBT}^{\cdot+} \mathrm{F} 4 \mathrm{TCNQ}{ }^{-}\right]+\mathrm{Li}^{+}+\mathrm{TFSI}^{-} \rightarrow\left[\mathrm{PNDTBT}^{++} \mathrm{TFSI}^{-}\right]+\mathrm{Li}^{+}+\mathrm{F} 4 \mathrm{TCNQ} \cdot$

Fig. 1 Summary of conjugated polymers used and anion-exchange doping. Chemical structures of a PNDTBT-C20 and b PNDTBT-4C16. Space-filling, van der Waals-based representations of $\mathbf{c}$ PNDTBT-C20 and $\mathbf{d}$ PNDTBT-4C16 with the dimer units illustrated for clarity. The characteristic void distance of the van der Waals surface between the alkyl side-chains is defined as $d_{\text {void. }}$. The error bars in $d_{\text {void }}$ stem from the uncertainty in reading the van der Waals surfaces. e Schematic diagrams illustrating anion-exchange doping, for which a solid-state thin film of PNDTBT is immersed in a dopant solution. F4TCNQ and an $\left[\mathrm{Li}^{+} \mathrm{TFSI}^{-}\right]$salt are co-dissolved in $n$-butyl acetate. Molecular doping proceeds with an initiator molecule F4TCNQ via a charge transfer interaction. The F4TCNQ radical anions, being part of a solid-state donor-acceptor pair [PNDTBT ${ }^{+}{ }^{+} 4 T_{C N Q}{ }^{-}$], are replaced instantaneously by TFSI ${ }^{-}$via anion exchange ${ }^{18}$.

of PNDTBT with propyl side chains was simulated by DFT calculations. This monomer unit was then aligned along the $\pi$ stack direction with each polymer long axis being pushed aside by 3.7 $\AA$. This value has been used typically for thiophene-based, crystalline polymers $^{28}$. In addition, the highest occupied molecular orbital levels for the two PNDTBT polymers are identical, determined by the observation of identical ionization potentials for both polymers (see Supplementary Information Section 1.2). This means that both polymers should undergo very similar redox reactions during molecular doping apart from their steric effects.

To assess the doping efficiency with regard to the steric effects, comparisons of the electronic and structural properties were investigated comprehensively between PNDTBT-C20 and PNDTBT-4C16. The optical absorption spectra reveal that a neutral $\pi-\pi^{*}$ absorption of PNDTBT polymers centered at approximately $500 \mathrm{~nm}$ is bleached, and a broad absorption at the near-infrared regime emerges after anion-exchange doping (Fig. 3a, b), which is consistent with hole doping of polymers. The broad absorption, which emerges after doping, may originate from localized polarons ${ }^{29,30}$. A characteristic doublet originating from the F4TCNQ radical anion is not seen in the absorption spectra of thin films after anion exchange. The absence of F4TCNQ ${ }^{-}$- after anion exchange is further verified by Fourier transform infrared (FTIR) spectroscopy (Fig. 3c, d). The FTIR spectra of the anion-exchanged thin films do not show the peak assigned to the $\mathrm{C} \equiv \mathrm{N}$ stretching mode of the $\mathrm{F}_{\mathrm{TCNQ}}{ }^{-}$ $\left(2190 \mathrm{~cm}^{-1}\right)$, which is an indicative of efficient anion exchange $\left(\right.$ F4TCNQ $^{-} \rightarrow$ TFSI $^{-}$). Note that the FTIR spectroscopy may possibly identify the intermediate (either integer charge transfer state or charge transfer complex ${ }^{31,32}$ ). Because no apparent superposition of neutral and anionized F4TCNQ peaks is observed in FTIR signal for polymers doped merely with F4TCNQ, it can be inferred that the intermediate during anion exchange is likely to undergo integer charge transfer. However, in reality, the initiator F4TCNQ and the second anion TFSI are supplied in solution at the same time, and the first initiation and second anion exchange processes may proceed cooperatively. Clearly, further studies are needed to identify the intermediate. The conductivity of thin films after anion exchange was increased by a factor of 4 and 40 compared with doping solely with F4TCNQ (see the conductivity values in the inset of Fig. 3a, b), suggesting that the doping level was improved by the efficient anion exchange with the optimum salt $\left[\mathrm{Li}^{+} \mathrm{TFSI}^{-}\right]$.

The degree of hole doping is significantly different in terms of the alkyl side-chain density; the bleaching of neutral absorption and increase of broad absorption at the near-infrared regime are found to be significantly greater for PNDTBT-C20, suggesting that a higher doping level can be realized for PNDTBT-C20 than for PNDTBT-4C16. Note that the oscillator strength in two polymers are almost comparable because the conjugated core as well as its conformation for two polymers is found to be very similar 24,26 . This trend is consistent with the experimentally measured electrical conductivity $\sigma\left(\sigma=183 \mathrm{~S} \mathrm{~cm}^{-1}\right.$ for PNDTBT$\mathrm{C} 20$ and $\sigma=0.4 \mathrm{~S} \mathrm{~cm}^{-1}$ for PNDTBT-4C16 after anion-exchange doping). 

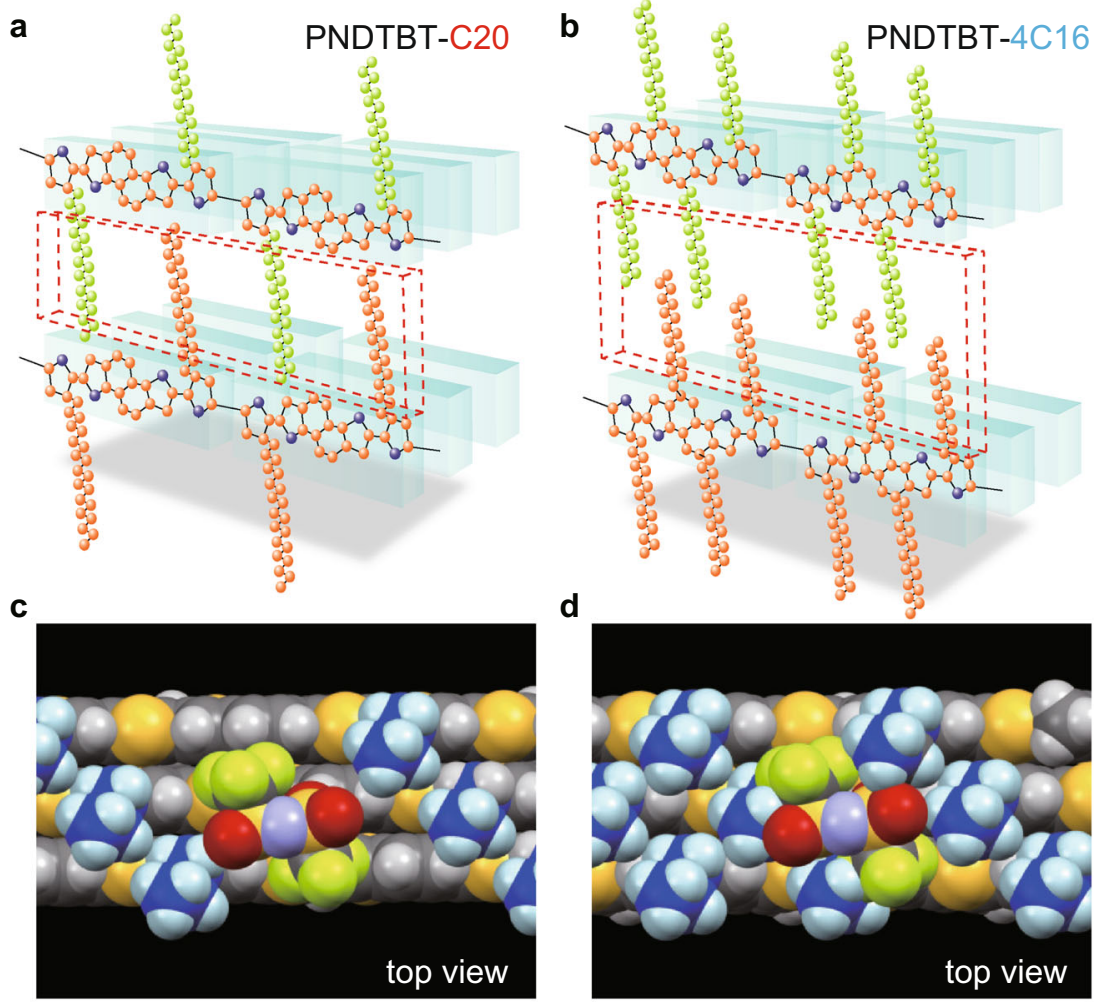

e

$\mathbf{f}$
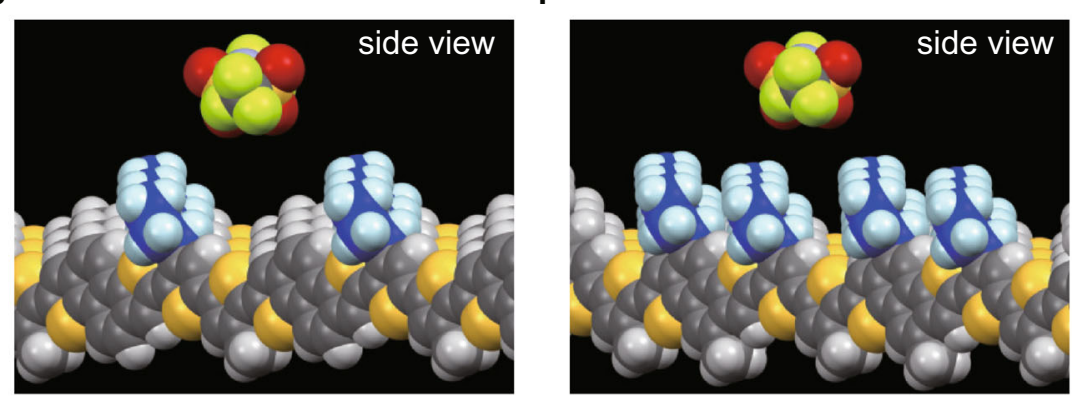

Fig. 2 Steric selectivity in molecular doping. Schematic illustrations of two-dimensional lamellar structure of a PNDTBT-C2O and $\mathbf{b}$ PNDTBT-4C16. The different color coding of $C$ atoms represents side chains from different layers (green symbols from the top layers, orange symbols from the bottom layers, respectively). Top-view space-filling, van der Waals-based illustrations of $\mathbf{c}$ PNDTBT-C20/TFSI and $\mathbf{d}$ PNDTBT-4C16/TFSI. Side-view illustrations of e PNDTBT-C20/TFSI and $\mathbf{f}$ PNDTBT-4C16/TFSI. The sphere representation of atoms is based on the hard cutoffs of van der Waals radii. The propyl sidechain located in the region capable of interacting with anions is shown in blue.

To address this difference, we investigated the host-guest hybrid structure by XRD measurements. The out-of-plane and inplane XRD profiles of a pristine (black) and a doped (colored) PNDTBT thin film are shown in Fig. 3e-h. The first-order (100) diffractions against the scattering vector $q_{z}$ are clearly observed for the pristine PNDTBT thin films regardless of side-chain density (Fig. 3e, f), indicating that a two-dimensional, lamellar structure is established for both pristine PNDTBT thin films. The observation of a well-ordered lamellar structure of PNDTBT polymers is consistent with previous literature ${ }^{26}$. The $d$-spacing is determined to be $23.4 \AA$ for PNDTBT-C20. This $d$-spacing is clearly shorter than the length of all-trans alkyl chain of C20 $(\sim 26 \AA)$, which suggests the possible side-chain inter-digitation ${ }^{26}$. The densely packed alkyl group has an effective diameter of about $5 \AA^{33}$, which is much shorter than the distance of the van der Waals surface between the alkyl side chains $\left(d_{\text {void }}\right)$ for the sparse PNDTBT-C20 (shown in Fig. 1c). On the other hand, $d_{\text {void }}$ for the dense PNDTBT-4C16 is about $4.5 \AA$ (shown in Fig. $1 \mathrm{~d}$ ), and is notably shorter than the effective diameter of the alkyl group.
This indicates almost no side-chain inter-digitation for PNDTBT4C16, which is consistent with the theoretical prediction ${ }^{20}$. A larger $d$-spacing obtained for PNDTBT-4C16 (30.2 $\AA$ ) is attributed to steric hindrance due to dense alkyl side-chains. We experimentally observe that the (100) diffractions are shifted in the small scattering vector direction for both PNDTBT thin films, which corresponds to an expansion of $d$-spacing. An $11 \%$ $d$-spacing expansion is obtained for doped PNDTBT-C20, whereas only a $4 \%$ expansion is obtained for doped PNDTBT4C16. The changes in the in-plane diffractions along $q_{x y}$ after doping also show different behaviors between PNDTBT-C20 and PNDTBT-4C16; the $\pi$-stacking distance, derived from the (010) diffraction peaks, decreases by $3 \%$ for doped PNDTBT-C20, whereas the (010) diffraction for doped PNDTBT-4C16 is hardly observable, or comparable to the noise level of the measurements. The overall XRD results suggest that TFSI ${ }^{-}$dopants introduced by anion exchange can intercalate into a restricted void space, namely the limited alkyl side-chain zone of PNDTBT polymers. The spatially confined intercalation causes the $d$-spacing 
a
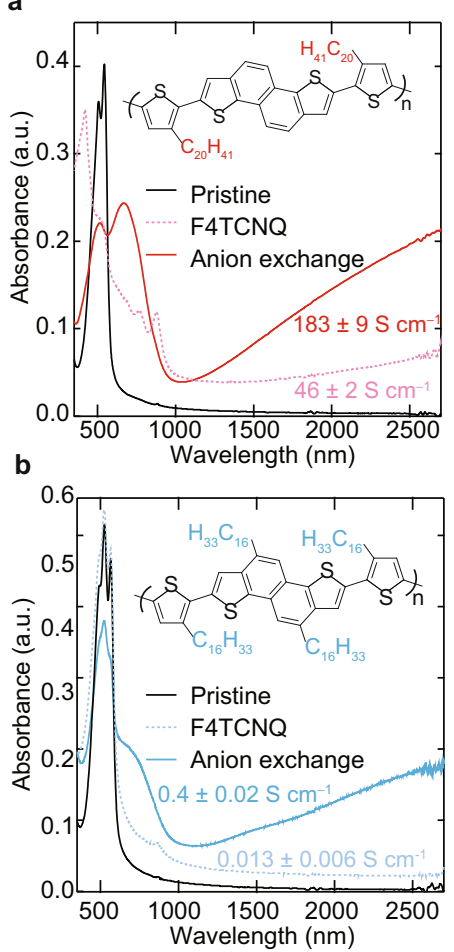

c

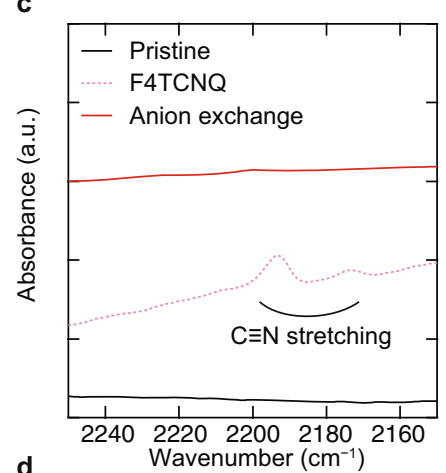

d

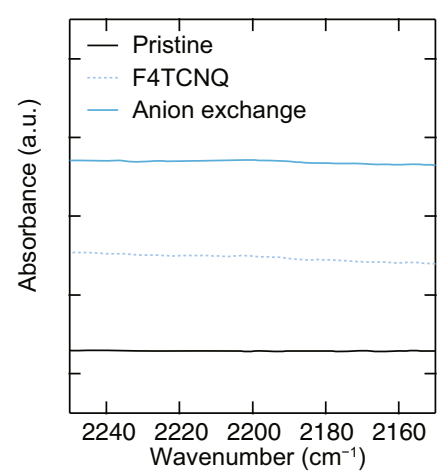

e

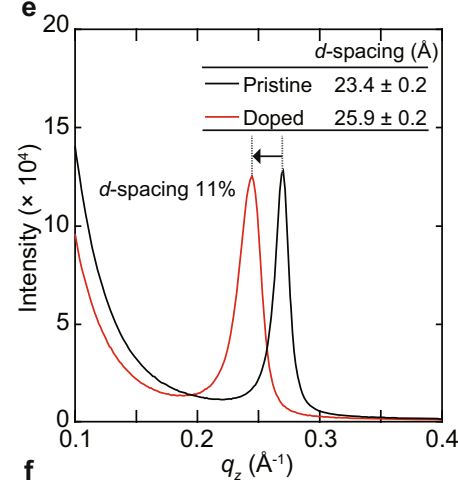

f

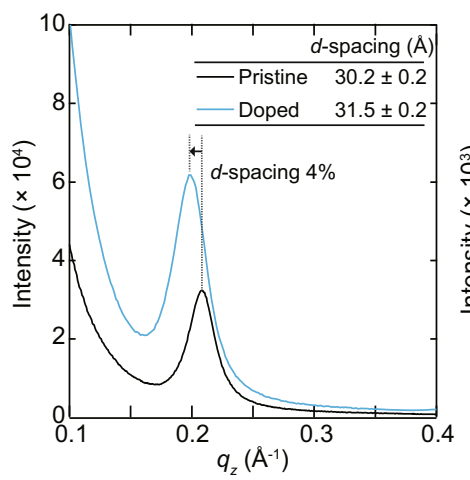

g
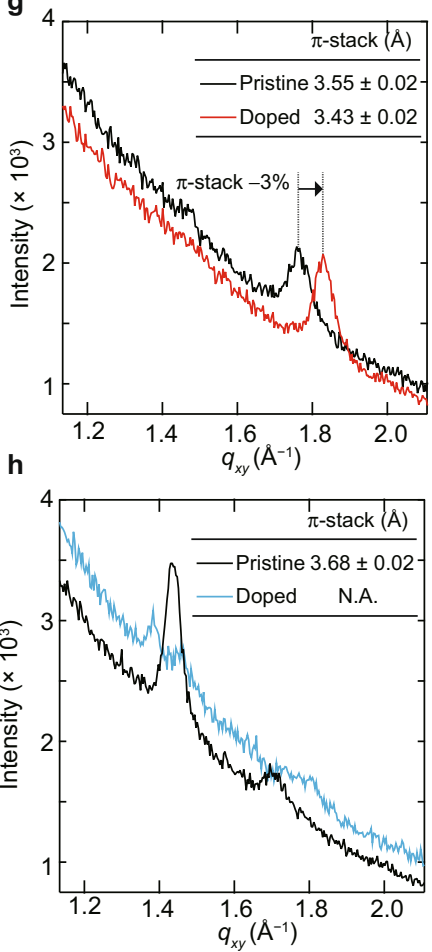

Fig. 3 Comparisons of doping efficiency. Optical absorbance spectra of a PNDTBT-C20 and b PNDTBT-4C16 before (black curve) and after anionexchange doping (colored solid curve). Data for thin films solely doped with F4TCNQ (dashed curve) are also plotted. The insets in both panels show the chemical structures of the polymers. The obtained electrical conductivities $\sigma$ are also shown in the insets. The error in the conductivity shows the uncertainty in the thickness of the polymer thin films, and represents one standard deviation. FTIR spectra of $\mathbf{c}$ PNDTBT-C20 and d PNDTBT-4C16 before (black curve) and after anion-exchange doping (colored solid curve). Data for thin films solely doped with F4TCNQ (dashed curve) are also plotted. Outof-plane X-ray diffraction profiles for e PNDTBT-C20 and f PNDTBT-4C16 before (black curve) and after anion-exchange doping (colored curve). The scattering vector along the out-of-plane direction of the films is defined as $q_{z}$. The obtained $d$-spacings are shown in the inset. In-plane X-ray diffraction profiles for $\mathbf{g}$ PNDTBT-C20 and $\mathbf{h}$ PNDTBT-4C16 before (black curve) and after anion-exchange doping (colored curve). The scattering vector across the in-plane direction of the films is defined as $q_{x y}$. The obtained $\pi$-stack distances are shown in the inset. The errors in the $d$-spacing and $\pi$-stack distance stem from uncertainties in fitting the diffraction peak, and represent one standard deviation.

expansion, which is consistent with the previous literature ${ }^{13-16,18}$. Because the void space formed between lamellae for PNDTBTC20 is clearly larger than that for PNDTBT-4C16, a large $d$-spacing expansion is expected for PNDTBT-C20, which agrees with the experimental results. Note that a decrease of the $\pi$-stack distance observed for PNDTBT-C20 can be attributed to a suppression of torsion of the PNDTBT backbone by doping ${ }^{18}$.

The steric selectivity found specifically in doped PNDTBT polymers is qualitatively understood by examining how TFSI $^{-}$is incorporated into the alkyl side-chain regime. It is found that PNDTBT-C20 can accommodate TFSI $^{-}$in its sparse alkyl sidechain zones without apparent short contact, whereas the incorporation of $\mathrm{TFSI}^{-}$can be limited by short contacts between the dense alkyl side-chains of PNDTBT-4C16 and TFSI ${ }^{-}$. Given that the molecular long axis of $\mathrm{TFSI}^{-}\left(d_{\text {long }}^{\mathrm{TFSI}}\right)$ is much greater than the void distance of the alkyl side-chains of PNDTBT-4C16 $\left(d_{\text {long }}^{\text {TFSI- }}=7.5 \AA>d_{\text {void }}=4.5 \AA\right)$, the short contact displayed in Fig. $2 \mathrm{~d}$ may be unavoidable. In a previous study ${ }^{18}$, we verified that anion exchange takes place spontaneously as long as the secondary donor-acceptor association reduces the Gibbs free energy. This situation can be achieved when the secondary donor-acceptor association has the optimum conformation. However, efficient anion exchange is no longer expected due to the presence of steric hindrance between alkyl side-chains of PNDTBT-4C16 and dopants, which limits the dopant incorporation and doping levels.
Formation of closest-packed supramolecules. From the observations above, we presume that PNDTBT-C20 is capable of accommodating a wider variety of functional anion dopants than has previously been considered. Particularly in the anion exchange process, the formation of an ion pair between a polymer and a large anion is likely to produce a large energy gain. This trend is intuitively understood by considering the empirical hard and soft acids and bases theory, which has been often invoked to explain ion exchange phenomena ${ }^{34}$. To verify the concept, we selected another anion tetrakis(pentafluorophenyl)borate (TFPB $^{-}$, shown in Fig. 4a, b). For DFT calculations, the molecular long axis of $\mathrm{TFPB}^{-}$is estimated to be $11.5 \AA$, and its electrostatic potential is found to be delocalized. This is because the electrostatic potential might be blurred due to the spread of the charge density to the phenyl group and the electron-withdrawing fluorine substituent. Anion-exchange doping with $\mathrm{TFPB}^{-}$was performed with a mixed dopant solution (F4TCNQ and $\left[\mathrm{Li}^{+} \mathrm{TFPB}^{-}\right]$ethyl ether salt co-dissolved in 1,2-dichloroethane). As expected, efficient anion-exchange doping was verified by observing the bleaching of the neutral absorption of PNDTBTC20 (Fig. 4c) and absence of F4TCNQ ${ }^{-}$in FTIR spectra (the inset of Fig. 4c). The electrical conductivity obtained for PNDTBT-C20 with TFPB $^{-}$is increased by a factor of 2.5 compared with that with $\mathrm{TFSI}^{-}$. In addition, the incorporation of bulky $\mathrm{TFPB}^{-}$is further confirmed by out-of-plane XRD measurements (Fig. 4d); the characteristic $d$-spacing is expanded by 
<smiles>O=C(NC(=O)C(F)(F)F)C(=O)NC(=O)C(F)(F)C(F)(F)F</smiles>

b

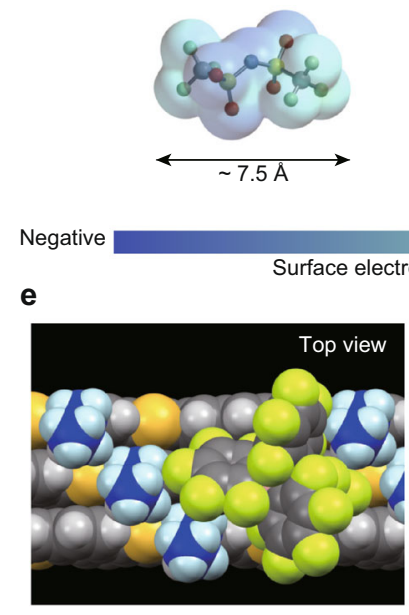

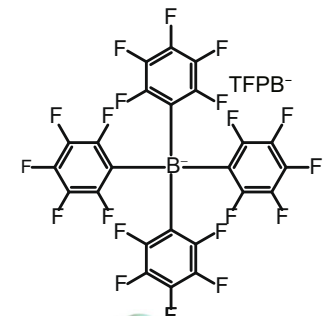

$F$

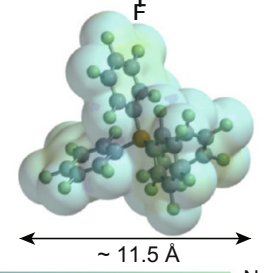

$f$

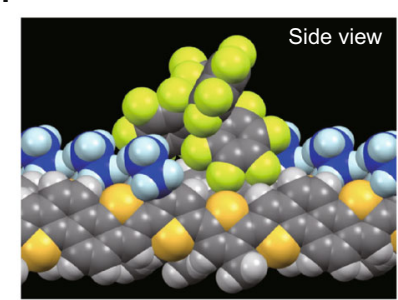

c

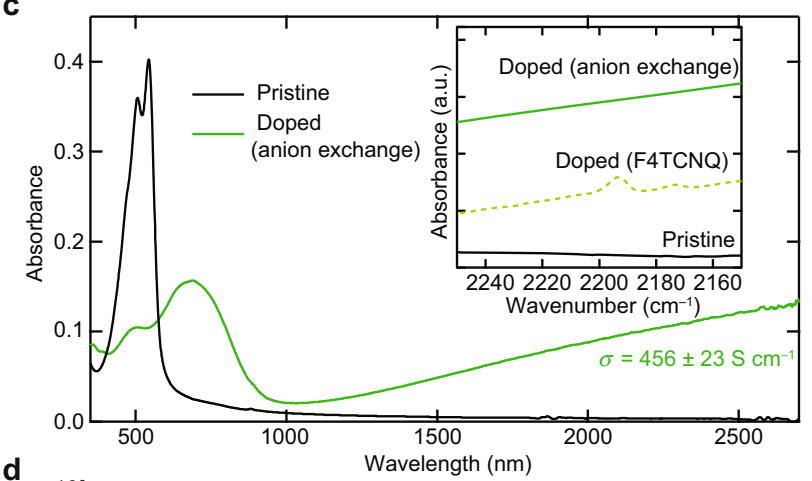

d

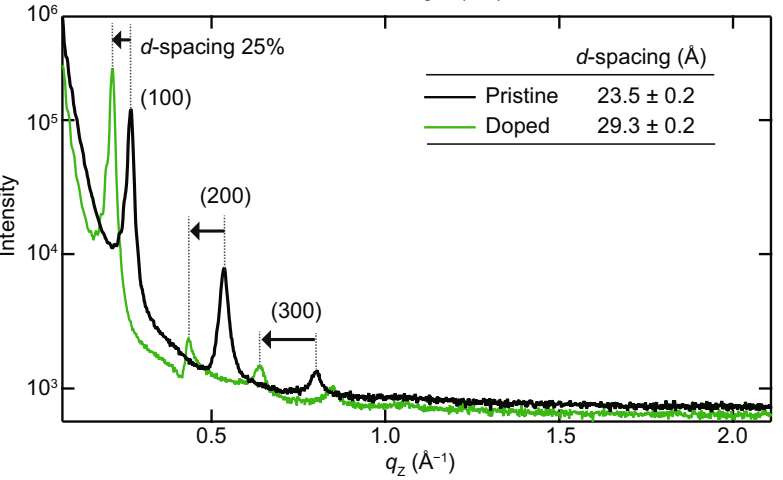

Fig. 4 Example of introduction of sterically bulky anions. a Molecular structures of $\mathrm{TFSI}^{-}$and $\mathrm{TFPB}^{-}$. $\mathbf{b}$ Electrostatic potential maps of the anions used. These calculated electrostatic potentials are based on the van der Waals surfaces of the anions. The spatial distributions of the electrostatic potentials were calculated using DFT with the B3LYP functional and 6-31+G(d) basis set (Spartan'16 software). c Optical absorption spectra of pristine PNDTBT-C20 (black) and of PNDTBT-C20 anion-exchange doped with TFPB- (green). The inset shows FTIR spectra of pristine PNDTBT-C20 (black curve) and of PNDTBT-C20 anion-exchange doped with TFPB- (green solid curve). Data for thin films solely doped with F4TCNQ (dashed curve) are also plotted. The obtained electrical conductivity $\sigma$ is shown in the inset. The error in the conductivity shows the uncertainty in the thickness of the polymer thin films, and represents one standard deviation. d Out-of-plane X-ray diffraction profiles for pristine PNDTBT-C20 (black) and for PNDTBT-C20 anion-exchange doped with TFPB- (green). The obtained $d$-spacings are shown in the inset. The error bars in the $d$-spacing stem from uncertainties in fitting the diffraction peak, and represent one standard deviation. e Top- and $\mathbf{f}$ side-view space-filling, van der Waals-based representations of PNDTBT-C20/TFPB.

$25 \%$ after doping with $\mathrm{TFPB}^{-}$, which is much larger than the $d$ spacing expansion obtained for PNDTBT-C20 with $\mathrm{TFSI}^{-}$. Schematics of space-filling representations of PNDTBT-C20/ TFPB show that even the bulky TFPB $^{-}$can be accommodated within the alkyl side-chain regions with no apparent short contact (Fig. 4e, f). Note that we also examined the introduction of TFPB to the most representative thiophene-based polymer, poly[2,5-bis (3-tetradecylthiophen-2-yl)thieno[3,2-b]thiophene] (PBTTT). However, we found that anion-exchange doping with TFPB ${ }^{-}$ never improves the doping levels because there is likely to be a short contact between alkyl side-chains of PBTTT and TFPB(see Supplementary Information Section 1.3). Overall, the investigation shows $\pi$-core extension in PNDTBT-C20, resulting in widening of the void space, which can facilitate the accommodation of a wide variety of functional dopants, and such controlled steric selectivity may be a key for developing further improvements of molecular doping.

The increase in conductivity for the bulky anion $\mathrm{TFPB}^{-}$was further investigated by magnetotransport measurements. The Hall effect is a powerful tool for determining charge carrier density and mobility separately, and also for assessing whether charge carriers undergo coherent, band transport, because the Hall voltage is only observed when the charge carriers undergo a free-electron-like transport. We observed a clear transverse (Hall) voltage using a Hall bar geometry (Fig. 5a, see more details in Supplementary Information Section 1.2). The symmetry and sign of the voltage correspond to the hole carrier conduction. From the obtained Hall voltage, the Hall carrier density $n_{\mathrm{H}}$ can be derived from the inverse Hall coefficient $n_{\mathrm{H}}=\left(e R_{\mathrm{H}}\right)^{-1}$, and the Hall mobility $\mu_{\mathrm{H}}$ can be determined from $\mu_{\mathrm{H}}=R_{\mathrm{H}} \sigma$. Comparisons of $n_{\mathrm{H}}$ and $\mu_{\mathrm{H}}$ between PNDTBT-C20 thin films anionexchange doped with $\mathrm{TFPB}^{-}$and with $\mathrm{TFSI}^{-}$are summarized in Fig. 5b, c. The doping density (hole per monomer unit) was estimated from the Hall carrier density and unit monomer density. In the previous literature ${ }^{24,26}$, the unit cell volume of PNDTBT polymer was determined to be $1.49 \times 10^{21} \mathrm{~cm}^{-3}$, equivalently monomer density of $6.71 \times 10^{20} \mathrm{~cm}^{-3}$. With the experimentally determined Hall carrier density of $6.8 \times 10^{20}$ and $8.5 \times 10^{20} \mathrm{~cm}^{-3}$, the doping density is estimated to be 1.0 and 1.3 hole per monomer unit for PNDTBT-C20 with TFSI and with TFPB, respectively. The results showed that the increased conductivity with $\mathrm{TFPB}^{-}$can be attributed to both the increased Hall carrier density and Hall mobility. The latter originates clearly from the improved crystallinity shown in Fig. 4d. A remarkably high carrier density corresponding to one hole per monomer unit achieved for PNDTBT-C20/TFPB is further confirmed by photoelectron yield spectroscopy. Figure $5 \mathrm{~d}$ plots the photoemission yield as a function of the incident photon energy, from which the ionization potential, equivalent to the effective Fermi energy, is monitored. We observed a larger shift in the threshold photon energy for PNDTBT-C20 with TFPB. This suggests that the formation of the ion pairs [PNDTBT-C20 ${ }^{\circ} \mathrm{TFPB}^{-}$] produces a 

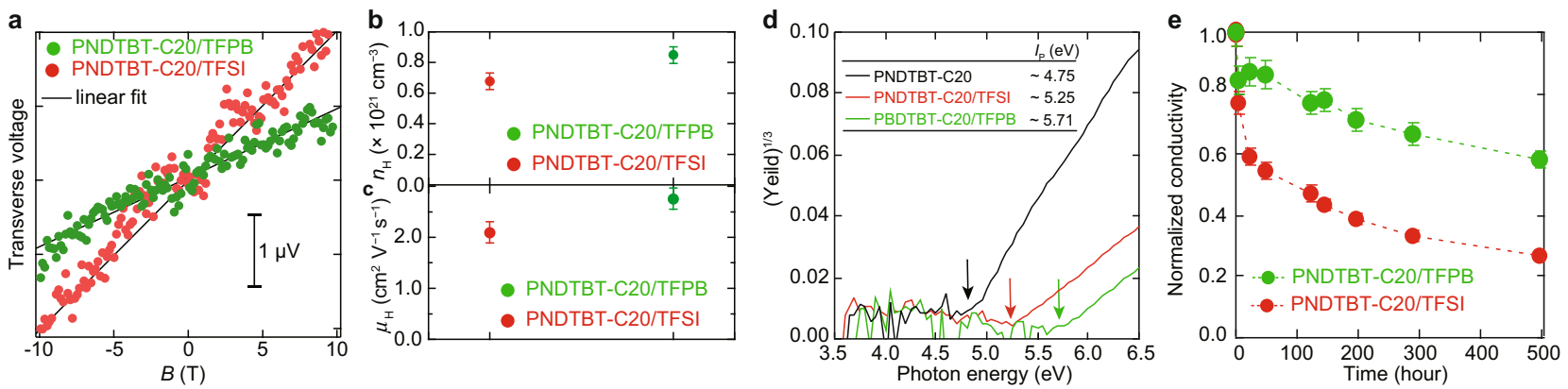

Fig. 5 Magnetotransport measurements and environmental stability. a Transverse (Hall) voltage against applied magnetic field $B$ for a PNDTBT-C2O thin film anion-exchange doped with TFPB- (green) and with TFSI- (red) at $240 \mathrm{~K}$. The measurements were conducted while $B$ was ramped up to $10 \mathrm{~T}$ and down to $-10 \mathrm{~T}$, where $B$ was applied perpendicular to the substrate plane. $\mathbf{b}$ Hall carrier density $n_{H}$ derived from the inverse Hall coefficient $n_{H}=\left(e R_{H}\right)^{-1}$ and $\mathbf{c}$ Hall mobility $\mu_{\mathrm{H}}$ estimated from $\mu_{\mathrm{H}}=R_{\mathrm{H}} \sigma$, for a PNDTBT-C20 thin film anion-exchange doped with TFPB- (green) and with TFSI- (red) at $240 \mathrm{~K}^{-}$ The error bars in $n_{\mathrm{H}}$ and $\mu_{\mathrm{H}}$ represent uncertainties in extracting the Hall voltage from the fitting and in the thickness of the polymer thin films, and represent one standard deviation. The Hall voltage was not detectable for the pristine PNDTBT-C2O because the the sample was highly resistive (the conductivity of pristine polymers was one the order of $\left.10^{-5} \mathrm{~S} \mathrm{~cm}^{-1}\right)$. d Photoemission yield spectroscopy results acquired from pristine PNDTBT-C20 (black), PNDTBT-C20/TFSI (red), and PNDTBT-C20/TFPB (green). The arrows represent the photon energy threshold corresponding to the ionization potential $I_{P}$. Values of $I_{P}$ are shown in the inset. e Environmental stability tests taken under ambient conditions. Electrical conductivities for PNDTBT-C20/ TFSI (red) and PNDTBT-C20/TFPB (green). The error bars in the conductivity show the uncertainty in the thickness of the polymer thin films, and represent one standard deviation.

larger gain of Gibbs free energy compared to the pairs [PNDTBT$\left.\mathrm{C}^{\circ} 0^{+} \mathrm{TFSI}^{-}\right]$, which is consistent with the expectation from the empirical hard and soft acids and bases theory tendency, where a soft cation is likely to form a pair with a soft anion ${ }^{18,34}$.

The incorporation of a soft anion with an extended electrostatic potential, such as TFPB $^{-}$, can also affect the environmental stability of molecular doping. Figure 5e plots the normalized conductivity against storing time in ambient conditions. PNDTBT-C20 polymers doped with TFPB and TFSI were fabricated with the similar manner at the same time, and were kept in an identical environment. Note that devices were kept in a clean-room condition, where the temperature and humidity are controlled to be $300 \mathrm{~K}$, and $65 \%$. These devices were kept at the same time and conductivity measurements were performed on the same period of time. Although a reduction in conductivity is observed in two samples, to different degrees, the reduction for PNDTBT-C20/TFPB is found to be suppressed markedly compared to PNDTBT-C20/TFSI. Conductivity degradation in the present host-guest system would be expected to occur only when anions react with other chemical species, such as a reaction with water (resulting in protonation). Thus, the hydrophobicity and chemical stability of anions are both important aspects in terms of improving environmental stability. The increased environmental stability we have observed can be attributed to the suppression of chemical reactions due to the incorporation of relatively large, soft anions. Anion-exchange doping in conjunction with molecular engineering of the void space, demonstrated in the present study, could accelerate further developments in overcoming redox potential limitations in molecular doping and improving the physicochemical properties of supramolecular conductors by tuning steric selectivity.

Our results show that steric effects between a $\pi$-conjugated semiconducting polymer and dopants could strongly influence the resulting donor-acceptor formation, which strongly limits the maximum achievable doping level in polymeric semiconductors. A closest-packed supramolecule is realized for a particular combination of a sparse conjugated polymer and an extraordinarily large dopant anion, which gives rise to high conductivity, high air stability, and a remarkably high work function. This work provides a critical insight into how to overcome steric effects in molecular doping, and highlights the importance of molecular engineering for further improvements of doping efficiency.

\section{Methods}

Materials. A family of naphthodithiophene-bithiophene polymers with different alkyl side-chain densities, namely PNDTBT-C20 with a sparse density and PNDTBT-4C16 with a high density, were synthesized and purified in house. The molecular weight $M_{\mathrm{w}}$ and polydispersity index (PDI) were estimated to be $59.95 \mathrm{kDa}$ and 1.46 for PNDTBT-C20 and $47.8 \mathrm{kDa}$ and 1.76 for PNDTBT-4C16 (see more details in Supplementary Information Section 1.1). Polymer thin films were deposited via spin-coating from $o$-dichlorobenzene solutions. During the spin-coating process, the sample chuck was heated at $75^{\circ} \mathrm{C}$ using a hot spin coater system. The resulting films were annealed on a hotplate at $200^{\circ} \mathrm{C}$ for $30 \mathrm{~min}$, and then slowly cooled to room temperature. The film thicknesses were determined to be $46 \pm 2 \mathrm{~nm}$ for PNDTBT-C20 and $51 \pm 2 \mathrm{~nm}$ for PNDTBT-4C16 using a Dektak surface profilometer and atomic force microscopy measurements. Anion-exchange doping was performed by immersing solid-state thin films into dopant solutions at $60{ }^{\circ} \mathrm{C}$ for $10 \mathrm{~min}$ in $\mathrm{N}_{2}$-purged vials. The concentration of dopants and the employed solvents are listed in Supplementary Table S1. After being removed from the vial, the films were annealed at $80^{\circ} \mathrm{C}$ for $10 \mathrm{~min}$, and the residual solvent was blown off under a flow of $\mathrm{N}_{2}$.

Transport measurements. Conductivity data were collected with a two-terminal device configuration. Doped polymer thin films were deposited onto $\mathrm{Cr}(3 \mathrm{~nm})$ and $\mathrm{Au}(30 \mathrm{~nm})$ electrodes, and then patterned into a rectangular shape to avoid the fringe effect that causes an overestimation of conductivity. For conductivity measurements, three devices with different channel lengths were tested. Hall effect measurements were performed using a Hall bar geometry, where the doped PNDTBT thin film was patterned by dry etching to allow precise measurements of the local potentials of probes along a channel. $\mathrm{Cr}(3 \mathrm{~nm})$ and $\mathrm{Au}(30 \mathrm{~nm})$ electrodes were thermally evaporated through a shadow mask on a pre-cleaned glass substrate. The PNDTBT thin film was deposited in a similar manner, and then patterned into the Hall bar geometry by laser etching using an yttrium aluminum garnet laser. Hall effect measurements were performed using an He gas exchanged cryostat with a superconducting magnet. Both the longitudinal and transverse electromotive forces were monitored simultaneously while applying a dc current of $1 \mu \mathrm{A}$ and sweeping the magnetic field from 10 to $-10 \mathrm{~T}$ at a rate of $0.4 \mathrm{~T} \mathrm{~min}^{-1}$.

DFT calculations. DFT calculations were conducted to visualize the van der Waals surface of PNDTBT monomer and dopant anions. Initially, the conformation of a monomer unit of polymers with propyl side-chains was simulated through structural optimization by DFT calculations, i.e., the monomer structure was energetically minimized (using the B3LYP functional and $6-31+\mathrm{G}(\mathrm{d})$ basis set). Note that the conformation of alkyl side chains impacts on steric selectivity, but it is known from the literature ${ }^{26}$ that alkyl side chains attached thiophene unit are likely to form all-trans conformation. Thus, replacing long side chains with the propyl group might be reasonable for qualitatively understanding steric effects in the present system. To construct a realistic molecular assembly of polymers, this monomer unit was then aligned along the $\pi$-stack direction with each polymer long 
axis being pushed aside by $3.7 \AA$. This value has been used typically for thiophenebased, crystalline polymers ${ }^{28}$. Finally, dopant anions were placed close to void spaces merely for visualizing whether the polymers' void space can accommodate dopant anions without short contact.

\section{Data availability}

The data that support the plots within this paper and other findings of this study are available from the corresponding author (S.W.; swatanabe@edu.k.u-tokyo.ac.jp) upon reasonable request.

Received: 1 May 2020; Accepted: 29 September 2020;

Published online: 04 November 2020

\section{References}

1. Sze, S. M. \& Ng, K. K. Physics of Semiconductor Devices (John Wiley \& Sons, 2006).

2. Bean, K. E. \& Runyan, W. Semiconductor Integrated Circuit Processing Technology (Addison-Wesley, 1990).

3. Shirakawa, H., Louis, E. J., MacDiarmid, A. G., Chiang, C. K. \& Heeger, A. J. Synthesis of electrically conducting organic polymers: halogen derivatives of polyacetylene, $(\mathrm{CH})_{\mathrm{x}}$. J. Chem. Soc. Chem. Commun. 578-580 (1977).

4. Heeger, A. J., Kivelson, S., Schrieffer, J. \& Su, W.-P. Solitons in conducting polymers. Rev. Mod. Phys. 60, 781 (1988).

5. Kar, P. Doping in Conjugated Polymers (John Wiley \& Sons, 2013).

6. Lüssem, B., Riede, M. \& Leo, K. Doping of organic semiconductors. Physica Status Solidi A 210, 9-43 (2013).

7. Salzmann, I., Heimel, G., Oehzelt, M., Winkler, S. \& Koch, N. Molecular electrical doping of organic semiconductors: fundamental mechanisms and emerging dopant design rules. Acc. Chem. Res. 49, 370-378 (2016).

8. Jacobs, I. E. \& Moulé, A. J. Controlling molecular doping in organic semiconductors. Adv. Mater. 29, 1703063 (2017).

9. Tang, C. G. et al. Doped polymer semiconductors with ultrahigh and ultralow work functions for ohmic contacts. Nature 539, 536 (2016).

10. Png, R.-Q. et al. Madelung and Hubbard interactions in polaron band model of doped organic semiconductors. Nat. Commun. 7, 1-9 (2016).

11. Tan, J.-K., Png, R.-Q., Zhao, C. \& Ho, P. K. Ohmic transition at contacts key to maximizing fill factor and performance of organic solar cells. Nat. Commun. 9, 3269 (2018).

12. Lin, X. et al. Beating the thermodynamic limit with photo-activation of $\mathrm{n}$ doping in organic semiconductors. Nat. Mater. 16, 1209-1215 (2017).

13. Kang, $\mathrm{K}$. et al. $2 \mathrm{D}$ coherent charge transport in highly ordered conducting polymers doped by solid state diffusion. Nat. Mater. 15, 896 (2016).

14. Fujimoto, R. et al. Control of molecular doping in conjugated polymers by thermal annealing. Org. Electron. 47, 139-146 (2017)

15. Fujimoto, R. et al. Molecular doping in organic semiconductors: fully solutionprocessed, vacuum-free doping with metal-organic complexes in an orthogonal solvent. J. Mater. Chem. C 5, 12023-12030 (2017).

16. Kim, Y. et al. Enhanced charge injection properties of organic field-effect transistor by molecular implantation doping. Adv. Mater. 31, 1806697 (2019).

17. Kiefer, D. et al. Double doping of conjugated polymers with monomer molecular dopants. Nat. Mater. 18, 149-155 (2019).

18. Yamashita, Y. et al. Efficient molecular doping of polymeric semiconductors driven by anion exchange. Nature 572, 634-638 (2019).

19. Watanabe, S. et al. Validity of the Mott formula and the origin of thermopower in $\pi$-conjugated semicrystalline polymers. Phys. Rev. B 100, 241201 (2019).

20. Kline, R. J. et al. Critical role of side-chain attachment density on the order and device performance of polythiophenes. Macromolecules 40, 7960-7965 (2007).

21. Lei, T., Dou, J.-H. \& Pei, J. Influence of alkyl chain branching positions on the hole mobilities of polymer thin-film transistors. Adv. Mater. 24, 6457-6461 (2012).

22. Miller, N. C. et al. Use of X-ray diffraction, molecular simulations, and spectroscopy to determine the molecular packing in a polymer-fullerene bimolecular crystal. Adv. Mater. 24, 6071-6079 (2012).

23. Osaka, I., Abe, T., Shinamura, S., Miyazaki, E. \& Takimiya, K. High-mobility semiconducting naphthodithiophene copolymers. J. Am. Chem. Soc. 132, 5000-5001 (2010).

24. Osaka, I., Abe, T., Shinamura, S. \& Takimiya, K. Impact of isomeric structures on transistor performances in naphthodithiophene semiconducting polymers. J. Am. Chem. Soc. 133, 6852-6860 (2011).
25. Osaka, I., Shinamura, S., Abe, T. \& Takimiya, K. Naphthodithiophenes as building units for small molecules to polymers; a case study for in-depth understanding of structure-property relationships in organic semiconductors. J. Mater. Chem. C 1, 1297-1304 (2013).

26. Osaka, I. et al. Contrasting effect of alkylation on the ordering structure in isomeric naphthodithiophene-based polymers. Macromolecules 47, 3502-3510 (2014).

27. Marcus, R. A. Electron transfer reactions in chemistry. Theory and experiment. Rev. Mod. Phys. 65, 599 (1993).

28. Cho, E. et al. Three-dimensional packing structure and electronic properties of biaxially oriented poly (2, 5-bis (3-alkylthiophene-2-yl) thieno [3, 2-b] thiophene) films. J. Am. Chem. Soc. 134, 6177-6190 (2012).

29. Pingel, P. \& Neher, D. Comprehensive picture of p-type doping of P3HT with the molecular acceptor F4TCNQ. Phys. Rev. B 87, 115209 (2013).

30. Tietze, M. L. et al. Elementary steps in electrical doping of organic semiconductors. Nat. Commun. 9, 1-9 (2018).

31. Neelamraju, B., Watts, K. E., Pemberton, J. E. \& Ratcliff, E. L. Correlation of coexistent charge transfer states in F4TCNQ-doped P3HT with microstructure. J. Phys. Chem. Lett. 9, 6871-6877 (2018).

32. Di Nuzzo, D. et al. How intermolecular geometrical disorder affects the molecular doping of donor-acceptor copolymers. Nat. Commun. 6, 1-8 (2015).

33. Yamamoto, T. et al. Extensive studies on $\pi$-stacking of poly (3-alkylthiophene2, 5-diyl) s and poly (4-alkylthiazole-2, 5-diyl) s by optical spectroscopy, NMR analysis, light scattering analysis, and X-ray crystallography. J. Am. Chem. Soc. 120, 2047-2058 (1998).

34. Pearson, R. G. Hard and soft acids and bases-the evolution of a chemical concept. Coord. Chem. Rev. 100, 403-425 (1990).

\section{Acknowledgements}

S.W. was supported by a Grant-in-Aid for JSPS (Japan Society for the Promotion of Science) Research Fellows. S.W. acknowledges assistance from PRESTO-JST "Hypernano-space design toward Innovative Functionality (Grant No. JPMJPR151E)”. This work was partly supported by a KAKENHI Grant-in-Aid (No. 15H05455) from JSPS.

\section{Author contributions}

Y.Y., S.K., and S.W. conceived and designed the experiments. S.K. and Y.Y. performed the measurements and analyzed the data. N.K. assisted with the magnetotransport measurements. T.M., I.O., and K.T. synthesized and purified the PNDTBT polymers. S.K and S.W. wrote the manuscript. J.T. and S.W. supervised the work. All the authors discussed the results and reviewed the manuscript.

\section{Competing interests}

The authors declare no competing interests.

\section{Additional information}

Supplementary information is available for this paper at https://doi.org/10.1038/s43246020-00081-3.

Correspondence and requests for materials should be addressed to S.W.

Reprints and permission information is available at http://www.nature.com/reprints

Publisher's note Springer Nature remains neutral with regard to jurisdictional claims in published maps and institutional affiliations.

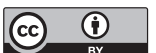

Open Access This article is licensed under a Creative Commons Attribution 4.0 International License, which permits use, sharing, adaptation, distribution and reproduction in any medium or format, as long as you give appropriate credit to the original author(s) and the source, provide a link to the Creative Commons license, and indicate if changes were made. The images or other third party material in this article are included in the article's Creative Commons license, unless indicated otherwise in a credit line to the material. If material is not included in the article's Creative Commons license and your intended use is not permitted by statutory regulation or exceeds the permitted use, you will need to obtain permission directly from the copyright holder. To view a copy of this license, visit http://creativecommons.org/ licenses/by/4.0/.

(C) The Author(s) 2020 